\title{
Vladimir Voevodsky
}

\section{Charles Weibel}

The mathematical community lost one of its brightest stars recently, when Vladimir Voevodsky passed away at the age of 51. In addition to being awarded the Fields Medal in 2002, he created two new areas of mathematics: motivic homotopy theory (a cross between topology and algebraic geometry), and an axiomatic formalization of mathematics, called univalent foundations.

The citation for his 2002 Fields Medal states that it was for his proof of the Milnor Conjectures, and for his concomitant development of motivic cohomology and motivic homotopy theory. He was also a member of the European Academy of Sciences, since 2003.

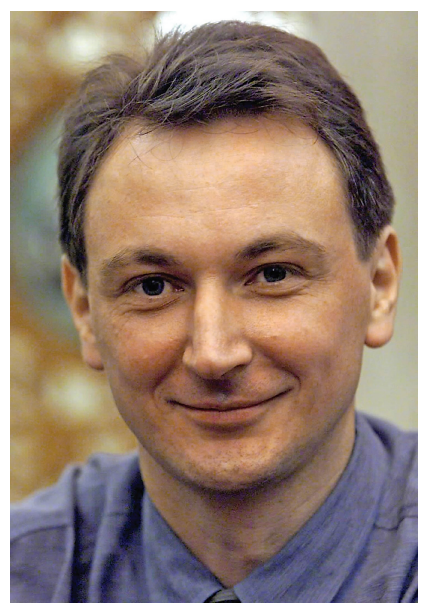

Vladimir Voevodsky (1966-2017).

\section{His Life}

Vladimir Alexandrovich Voevodsky was born in Moscow on June 4, 1966 and died in Princeton on September 30,

Charles Weibel is professor at Rutgers University. His email address is weibe1@math. rutgers . edu.

Communicated by Notices Associate Editor Della Dumbaugh.

For permission to reprint this article, please contact:

reprint-permission@ams . org.

DOI: https://doi.org/10.1090/noti1850
2017, at the age of 51. For the next two pages, I will concentrate on his (colorful) early career.

Voevodsky's parents were both scientists. His father, Alexander, directed a laboratory in experimental physics at the Russian Academy of Sciences; his mother, Tatyana Voevodskaya, was a chemistry professor at Moscow University. As a youth, Vladimir was kicked out of high school three times, once for disagreeing with his teacher's assertion that the author Dostoyevsky, who died in 1881, was pro-Communist.

In 1983, at the age of 17, he enrolled at Moscow State University. He soon became bored by his classes and stopped attending them several times, taking what one might call several "gap semesters." Finally, in 1989, he was expelled for what he called "academic failure."

As was common, Voevodsky had a day job: working at the Lycee of Informational Technologies, as a technician responsible for running and fixing the printers at the Computer Center. There he met Professor George Shabat, who was also working in the Computer Center. When Voevodsky asked to be unofficially mentored by him, Shabat assigned a difficult problem in order to rid himself of what appeared to be just another student. After a few days, Voevodsky returned with a solution-and several examples worked out on the computer. This led to a collaboration [ShV, ShV1] about aspects of Grothendieck's Dessins d'enfants, with the English version of [ShV1] entitled "Drawing Curves over Number Fields." While still officially a student, Voevodsky wrote three more papers [V90, V91, V91a] in this vein.

Another collaboration sprung up during Voevodsky's undergraduate days, with Mikhael Kapranov. This resulted in the papers [KV1, KV2, KV3, KV4] on homotopy types and what Ronnie Brown called " $\infty$-categories," structures defined using multi-simplicial sets.

At the same time, the Soviet Union was slowly collapsing. Gorbachev had introduced perestroika (the restructuring of the Soviet political and economic system) and glasnost (openness). The Berlin Wall fell in November 1989, 


\section{MEMORIAL TRIBUTE}

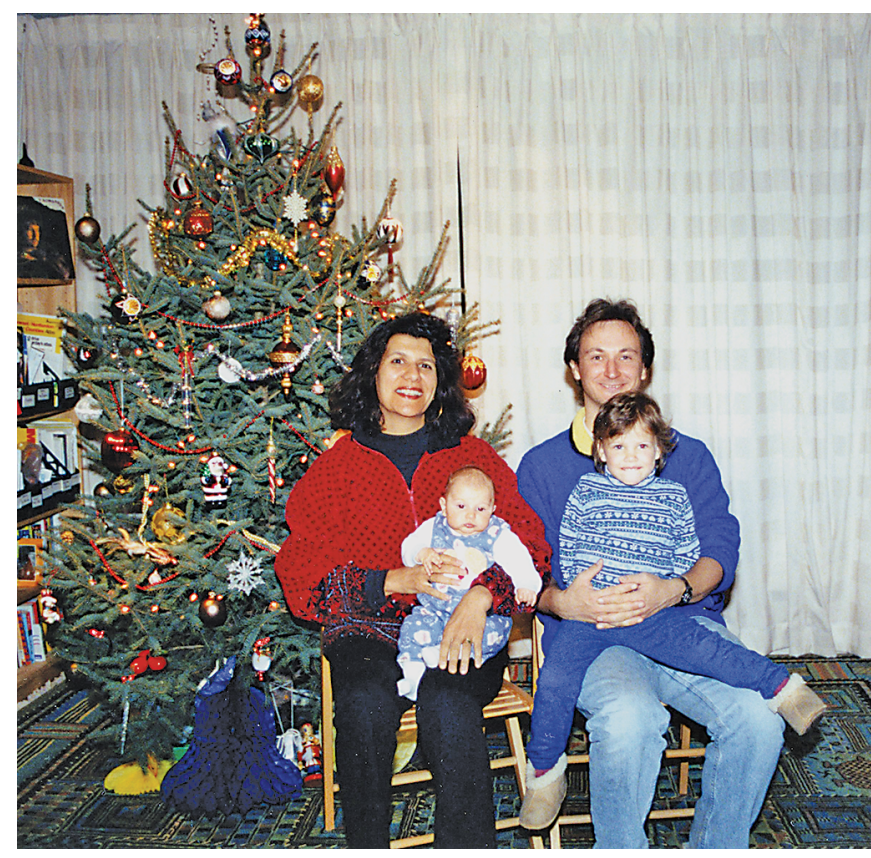

Voevodsky with his family (2001).

and the Soviet Union itself was in its last days. Travel abroad was becoming possible; in 1978, Margulis was not allowed to go to Helsinki to receive his Fields medal, but by 1989 it had become possible for Russians to enroll in a university abroad.

At this point, Voevodsky wanted to go to graduate school at the University of Wales, to work on category theory with Ronnie Brown. As an alternative, his friends Alexander Beilinson and Mikhail Kapranov arranged for him to be accepted at another university, one named Harvard, which Voevodsky had never heard of. After some persuasion, they convinced him to travel to the United States and attend Harvard.

Thus it was that Voevodsky became a graduate student at Harvard University in 1990, without a high school or college degree, and without even formally applying. Unsurprisingly, he had difficulty adjusting to life in Boston, and was even robbed at one point, so he temporarily went back to Russia. Upon returning, he lived in his office for a time. Nevertheless, he received his doctorate in 1992, under David Kazhdan. It is worth noting that this $\mathrm{PhD}$ was the only academic degree Voevodsky ever received!

In 1990, Vladimir met Nadia Shalaby, his life-long partner, at Harvard. They married in 1995 and had two children, Natalia Dalia Shalaby and Diana Yasmine Voevodsky, both of whom are now in college. Although the marriage ended in divorce in 2008, Vladimir and Nadia remained close friends all his life.

Upon graduating, Voevodsky spent a year at the Institute of Advanced Study (IAS), before returning to Harvard

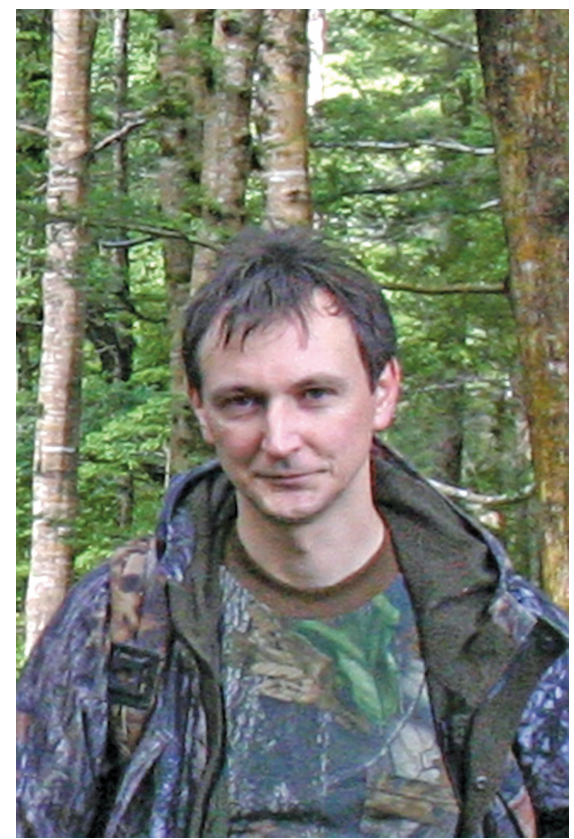

He loved walking in the woods and taking photographs of nature.

in 1993-96 as a junior fellow in the Harvard Society of Fellows. According to Eric Friedlander, Voevodsky complained about having to go to dinner occasionally as a junior fellow at Harvard, as it took time away from his research.

After a year at the Max-Planck Institute, Voevodsky joined the faculty at Northwestern University in 1997. Although he was a gifted teacher, he did not enjoy teaching_again for the reason that it kept him from his research. In 1998, he accepted a position as a long-term member at IAS, becoming a Professor (permanent member) of IAS in 2002. When he was appointed to the IAS, he said with relief that he wouldn't have to teach any more!

He remained a Professor at the Institute of Advanced Study for the rest of his life. On September 30, 2017, after Nadia Shalaby had failed to reach him by telephone, friends found him in his Princeton home, having collapsed in his home from a sudden aneurysm.

The Institute for Advanced Study held a Memorial Service for him a week later (on October 8 ), and a funeral service was held for him in Moscow on December 27, 2017, followed by a Memorial Mathematical Conference on December 28, 2017 at the Steklov Mathematical Institute of the Russian Academy of Sciences.

A year later, two memorial conferences were held simultaneously. One was held September 11-14, 2018, at the Institute for Advanced Study in Princeton; the other was held September 10-14, 2018 at the Euler International Mathematical Institute in St. Petersburg, Russia. 


\section{Voevodsky's Mathematics}

I now turn to Voevodsky's mathematical accomplishments. They are organized around several of his revolutionary ideas.

Motivic cohomology (1992-1995). Voevodsky's 1992 thesis, published as [V96], constructed a triangulated category $D M(S)$ for any noetherian base $S$, as an approximation to the hypothetical abelian category of "mixed motives" envisioned by Grothendieck, Deligne, Beilinson,

Lichtenbaum, and others. Any scheme $X$ over $S$ is represented by an object $\mathbb{Z}(X)$ in $D M(S)$, and the motivic cohomology of $X$ with coefficients in $F$ is defined as the Ext-groups $H^{n}(X, F)=\operatorname{Ext}^{n}(\mathbb{Z}(X), F)$.

When $S$ is the scheme associated to a field, the category $D M(S)$ in his thesis differs only in sophistication from the subcategory of effective motives in the category $D M$ that Voevodsky would use later; see [V00b]. Competing constructions were made by $\mathrm{M}$. Hanaumra and M. Levine at about the same time.

The paper [V96] contained three revolutionary ideas, ideas which Voevodsky would continue to develop over the next few years. One was to divide the problem of constructing a category of motives into two parts: (a) constructing a triangulated category $D M$ satisfying some basic properties, and (b) showing that $D M$ is the derived category of an abelian one. (He accomplished (a), but (b) remains an open problem.)

Another new idea was to work with sheaves on schemes over $S$ with respect to two new Grothendieck topologies: the $h$-topology and the quasi-finite $h$-topology (or $q f h$ topology). As Voevodsky remarked in [V96], the usual topologies (Zariski, étale, ...) "do not satisfy the properties we would expect from the 'theory of motives."

A third idea was to construct $D M$ from a derived category of sheaves $D$ with respect to the thick subcategory generated by the "contractible" objects $F \otimes \mathbb{A}^{1} \rightarrow F$, where (in this application) $\mathbb{A}^{1}$ is the affine line.

Almost immediately, he began working with Andrei Suslin in developing these ideas. In 1992, they used the af $h$ topology to construct a homology theory for schemes over an arbitrary field which, over $\mathbb{C}$ and with finite coefficients, agrees with the singular homology of the underlying topological space. This was published in 1996, as [SV96].

In 1994, Voevodsky released a series of foundational papers on motivic cohomology with Suslin and Eric Friedlander: [V00a, V00b, FV, SV00a, S00]. These were later published in book form in 2000 [VSF]. Around this time, Suslin showed that motivic cohomology agrees with the higher Chow groups defined by Bloch in [B86] in characteristic 0; see [MVW]; the characteristic 0 assumption was later removed in [V02a].
In 1995, he wrote a related paper [V95] about correspondences between smooth projective varieties. The rational equivalence classes of such correspondences form the morphisms in Grothendieck's category of Chow motives. Generalizing the observation that a correspondence from $X$ to itself that is algebraically equivalent to 0 is a nilpotent endomorphism of $X$, he formulated the notion of a correspondence $f: X \rightarrow Y$ being smash nilpotent: some $f^{\otimes n}: X^{\otimes n} \rightarrow Y^{\otimes n}$ is trivial. Then he stated the Nilpotence conjecture: A correspondence $f: X \rightarrow Y$ is smash nilpotent if and only if it is numerically equivalent to zero (that is, conjecturally, homologous to zero).

By 1995, Voevodsky was essentially finished with the framework of motivic cohomology. In 1999-2000, he gave a course on the subject at the Institute for Advanced Study, which my student Carlo Mazza and I attended. At the end of the course, Carlo and I were asked to write up the lecture notes. Whenever Carlo and I could not reconstruct an argument (often a proof that consisted of the word "obvious"), we would come to Voevodsky's office and he would explain it to us. This process uncovered a mistake in a key lemma [V00a, 4.23], which was corrected in [MVW, 22.10]. Thomas Geisser was also very helpful with some points. By 2004 we were finished, and the book [MVW] appeared in 2006.

$\mathbb{A}^{1}$-homotopy theory (1996-2000). The notion of an $\mathbb{A}^{1}$ homotopy theory for rings arose around 1970, largely due to the work of Steve Gersten [G]. The naive idea was that polynomials in $t$ should be regarded as homotopies between $t=0$ and $t=1$. Thus if $F$ is a functor from rings to sets, such as $G L_{n}$, two maps $f_{0}, f_{1}: F(R) \rightarrow$ $F(S)$ are considered "homotopic" if there is a map $f$ : $F(R) \rightarrow F(S[t])$ so that $f_{i}$ is the composite of $f$ with the map $F(S[t]) \rightarrow F(S)$ induced by $t \mapsto i$. Any $F$ has a universal homotopy invariant quotient: the coequalizer of $t=0,1: F(R[t]) \rightrightarrows F(R)$. However, it was quickly discovered that this naive definition of homotopy theory was useful only in very limited contexts, and did not generalize well from rings to varieties.

In September 1995, Voevodsky heard about the work of Fabien Morel, who was just finishing his Habilitation in Paris, and was trying to define a natural homotopy theory on algebraic varieties using Quillen's model categories. Having had similar ideas, Voevodsky began an email correspondence with Morel. In May 1996, they met in person and Voevodsky proposed that they "should write a bit." The result was their joint paper $\mathbb{A}^{1}$-homotopy theory of schemes [MV], released in 1998, which laid the foundations for what is (not surprisingly) called " $\mathbb{A}^{1}$-homotopy theory." A few months later, Voevodsky gave a beautiful address at the 1998 Berlin ICM [V98], laying out the foundations of stable $\mathbb{A}^{1}$-homotopy theory and motivic spectra. 
In 2000, Voevodsky defined the slice filtration on the stable motivic homotopy category defined in [V98]. The slices $s_{n} E$ of a motivic spectrum $E$ are again motivic spectra, and they form the motivic analogue of the Postnikov tower in classical stable homotopy theory. This tower yields a "slice" spectral sequence

$$
E_{2}^{p, q}=H^{p}\left(X, s_{-q}^{p+q, n} E\right) \Rightarrow E^{p+q, n}(X) .
$$

Voevodsky discussed several aspects of this filtration in [V02b], ending with a list of open conjectures. Voevodsky settled one in [V04], proving that the $0^{\text {th }}$ slice of the motivic sphere spectrum $E=\mathbb{S}$ is the object $H \mathbb{Z}$ that represents motivic cohomology. All of the other conjectures have since been confirmed over fields of characteristic 0 , but many remain open in finite characteristic.

The paper [V02c] studies the case when $E$ is the motivic spectrum $K G L$ representing algebraic K-theory. The resulting spectral sequence has $E_{2}^{p, q}=H^{p-q,-q}(X, \mathbb{Z})$ and converges to $K_{-p-q}(X)$. Suslin later showed that this spectral sequence may be identified with a spectral sequence first formulated by Bloch and Lichtenbaum.

A good introduction to stable $\mathbb{A}^{1}$-homotopy theory, including the slice filtration, is given by Voevodsky's notes [VRØ], based on a short course Voevodsky gave in 2002.

In 2000-1, Voevodsky gave a full-year course at IAS on equivariant motivic homotopy theory. The main application was in the action of the symmetric group $\Sigma_{n}$ on the product $X^{n}$, and its resulting equivariant motive. This material was later used to analyze symmetric powers $\operatorname{Sym}^{n} X$ of a variety $X$, which occur in the proof of the Bloch-Kato conjecture. Each week, Deligne would give Voevodsky his notes on the lecture, and Voevodsky typed it up. The result was published as [D].

The Milnor conjectures (1995-2000). Let $p$ be a prime, and let $F$ be a field of characteristic not $p$, containing the $p^{\text {th }}$ roots of unity for simplicity. Around 1969, Tate constructed the norm residue map from $K_{2}(F)$ to $H_{\mathrm{et}}^{2}(F, \mathbb{Z} / p)$, and Milnor defined abelian groups $K_{n}^{M}(F)$ so that the norm residue map generalized to a map $K_{n}^{M}(F) / p \rightarrow H_{\mathrm{et}}^{n}$

$(F, \mathbb{Z} / p)$. (Milnor's $K_{2}^{M}(F)$ agrees with $K_{2}(F)$, which is defined using the Steinberg group.) In 1970, focusing on the case $p=2$, Milnor stated, "I do not know of any examples for which the [norm residue] homomorphism fails to be bijective." [M70, p. 340]. The assertion that it is always an isomorphism became known as the Milnor Conjecture. It was established around 1982 by Merkurjev and Suslin in their celebrated paper [MS82] when $n=2$, for all $p$. Some results for $n=3$ were later established by Rost, Levine, and Merkurjev-Suslin.

In March 1996, Voevodsky announced a proof of the Milnor Conjecture; his preprint followed that December.
The following summer, he gave lectures on the proof at an AMS Summer Research Conference in Seattle. I was tasked with writing up the lecture notes, and they appeared in 1998 as [V99]. Preparing these notes let me really get to know Volodia, as he was very generous with his time in expaining the various aspects of the proof to me. The official proof, published in 2003 [V03a], differed in several places from the original preprint because Voevodsky had found a shorter method of proof.

In the same 1996 lecture that he announced a proof of the Milnor conjecture, Voevodsky also announced that he, Orlov, and Vishik had proven "Milnor's conjecture for quadratic forms." Formulated as "Question 4.3" in [M70], it asked if a certain map $s_{n}: K_{n}^{M}(F) / 2 \rightarrow I^{n} / I^{n+1}$ is an isomorphism for all $n$ and $F$. Here $I$ is the augmentation ideal of the Witt ring $W(k)$ of quadratic forms over a field $F$. Milnor showed that $s_{2}$ was an isomorphism; $s_{3}$ and $s_{4}$ were shown to be isomorphisms in the late 1980s by Rost and Merkurjev-Suslin, but the general case remained open until the preprint based on this announcement appeared in 1997; Vishik was Voevodsky's student and the preprint is cited in his thesis [Vsh]. The paper [OVV] was published in 2007.

The Bloch-Kato and Beilinson-Lichtenbaum conjectures. The analogue of the Milnor conjecture when $p$ is odd, dubbed the Bloch-Kato conjecture by Suslin, was first clearly formulated in 1980 by Kazuya Kato in [Kato, p. 608]:

Conjecture. The [norm residue] homomorphism is bijective for any field $k$ and any integer $p$ which is invertible in $k$.

Spencer Bloch's version was: "I wonder whether the whole cohomology algebra $\oplus_{r} H_{\mathrm{et}}^{r}\left(F, \mu_{p^{v}}^{\otimes r}\right)$ might not be generated by $H^{1 "}$ [B80, p. 5.12].

In a 1995 preprint, Suslin and Voevodsky showed that the Bloch-Kato conjecture is equivalent to a conjecture originally made by Lichtenbaum and modified by Beilinson to connect motivic cohomology groups to étale cohomology. More precisely, if $\pi$ is the usual morphism from the étale site to the Zariski site, the Beilinson-Lichtenbaum conjecture was that the motivic complex $\mathbb{Z} / p(n)$ should be quasi-isomorphic to the truncated complex $\tau_{\leq n} R \pi_{*}$ $\left(\mu_{p}^{\otimes r}\right)$. It is notable for its introduction and use of the $c d h$ topology. After substantial rewriting, this result appeared in 2000 as [SVOOb].

In 1998, Voevodsky announced a proof of the BlochKato conjecture, assuming the existence of what we now call a Rost variety. Rost produced such a variety that same year, in [Ro], but the complete proof that Rost's variety had the properties required by Voevodsky did not appear until 2007. An outline of Voevodsky's proof appeared in a 2003 preprint, modulo the assumption that Rost varieties exist and two other assertions. The second assertion, that every 


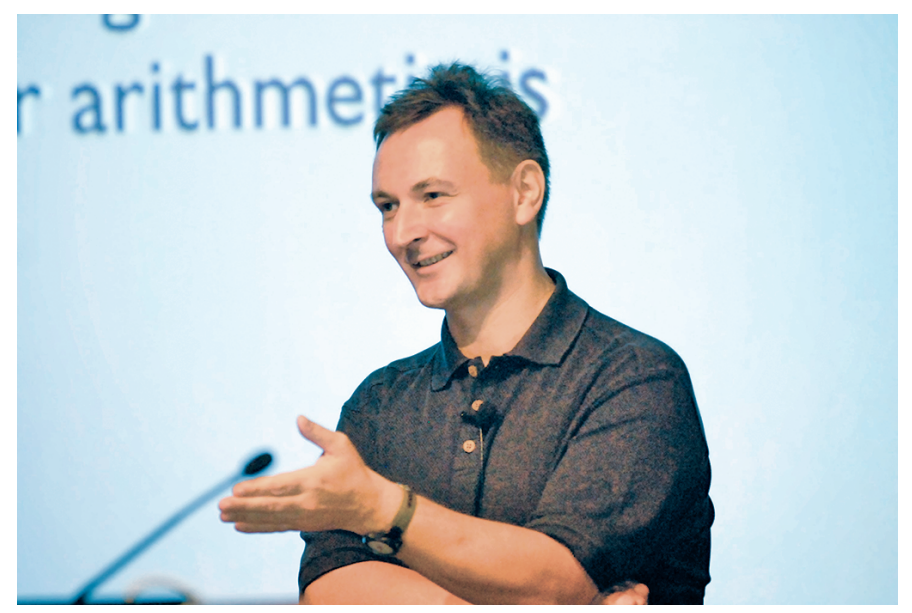

Voevodsky in 2010.

mod- $p$ motivic cohomology operation is a polynomial in the $P^{a}$, turned out to be wrong.

In 2006-7, I was asked to run a seminar at IAS, explaining the state of affairs of the Bloch-Kato conjecture. Voevodsky generously shared his source files with me, and I spent a lot of time talking to him about them. Although his second assertion was false, I saw that it was not really needed, and patched his proof by using the notion of scalar weights for cohomology operations [W09]. This re-engaged Voevodsky in the project and he produced the final part of the proof in 2008. The next few years saw a flurry of long-delayed publications appear: [V10a, V10b, V10c, V10d,V10e, V10f] and the final piece of the puzzle in [V11]. The complete proof will be published soon in the book [HW].

Other interests. Voevodsky was briefly involved in several other projects. For example, in 1997 he spent several months thinking about artificial intelligence in robotic locomotion.

In 2001-2, Voevodsky gave a full-year course at IAS on the formalism of the two adjoint pairs of functors $\left(f^{*}, f_{*}\right)$ and $\left(f_{!}, f^{!}\right)$associated to a morphism $f$ of schemes. Together with the adjunction of $\otimes$ and Hom, this amounts to a generalization of Grothendieck's "six-functor formalism." Although Voevodsky never published his results, Joseph Ayoub figured out the details and published them as [Ayb].

In 2003-2008, Voevodsky became interested in inferring genetic history from current data. At each time $t \geq-T$, there is a set of (male) genomes $X_{t}$. As $t$ varies we get a graph: it is a forest of valence 3 , vertices being births and deaths. If one assumes a constant birth rate, we would like to know the most likely values of $X_{-T}$ and death rates which would produce a given population $X_{0}$ at time 0 . Biologists refer to this process as "the coalescent." As he explained it to me, Voevodsky had the idea that births and deaths could be described by a Markov branching process. Since time is totally ordered, we may regard it as a category, and one regards $X$ as a stochastic category fibered over time. When he felt he was ready, he gave a lecture on this at Ohio State's Math Biosciences Institute; the lecture was not well received. Looking back in 2013, he said, "I wasted two years, because I totally failed." (See [Reh].)

In 2005-2006, Voevodsky was excited by what he called "homotopy $\lambda$-calculus" as a generalization of Church's $\lambda$ calculus of types. Working in the homotopy category of topological spaces (in a fixed universe), he inductively defined a nested sequence of "levels." Reindexing, $X$ has level 0 if it is contractible, level 1 if it is either the empty set or a contractible space, and $X$ has level 2 if it is homotopy equivalent to a discrete set; $X$ has level $n+1$ if the path spaces $P\left(X, x, x^{\prime}\right)$ have level $n$ for all pairs $X, x^{\prime}$ in $X$. Homotopy $\lambda$-calculus would later evolve into homotopy type theory, and the levels in homotopy $\lambda$-calculus were the prototypes of the " $h$-levels" in the Univalent Foundations. Type theory. The inadvertent mistake in [V00a], mentioned above, led Voevodsky to become interested in using computers to verify proofs. Much later, in a public lecture in 2014, he would state the truism that "a technical argument by a trusted author, which is hard to check and looks similar to arguments known to be correct, is hardly ever checked in detail."

Starting in 2004, he gradually focused on Per MartinLöf's formal language of "types" (and their elements) as a way to ensure grammatical correctness. The word type is an undefined term, much as the word set is an undefined term in Zermelo-Fraenkel set theory. One feature of this language is that if $x, x^{\prime}$ are elements of the same type then there is a new type: the type of "identifications" of $x$ and $x^{\prime}$. This led Awody and Bauer to define a proposition as a type whose elements are (pairwise) equal (see $[A w B]$ ); informally, all of its proofs are indistinguishable.

At this point, he started learning about "proof assistant" programs such as Coq. To learn how to use Coq, Voevodsky took an undergraduate course at Princeton in Fall 2009, from Andrew Appel, called "Programming Languages." The course used Coq for doing machine-checked proofs in logic, with application to the semantics of programming languages, type systems, and verifying the correctness of algorithms and programs. Voevodsky completed the midterm exam, which was verification of the correctness of the "Binomial Queues" data structure. A few years later, he told Appel that the exam "convinced him that machine-checked proof in Coq could be a practical way to do mathematics." 
Voevodsky integrated the computer into the process of doing his own research, describing it in a 2013 interview [Reh] as a bit like a video game. "You tell the computer, 'Try', and it tries, and it gives you back the result of its actions... Sometimes it's unexpected what comes out of it. It's fun."

At this point he embarked on an enormous project to create proof-checking software so powerful and convenient that mathematicians could someday use it as part of their ordinary work and create a library of rock-solid mathematical knowledge that anyone in the world could access. This library, called Foundations, is described in [V15b].

The Univalence axiom. Already in 2006, Voevodsky had the notion that there were special maps which he called "univalent." In late 2009, he wrote to Grayson that his ideas about a univalent homotopy-theoretic model of type systems had developed enough to "survive the verification stage, and I am in the process of writing things up." Voevodsky announced his new axiom for type theory, the univalence axiom, in a lecture he gave at Carnegie Mellon, early in 2010. Since then, this axiom has had a dramatic impact on both mathematics and computer science.

The univalence axiom states that

$$
(X=Y) \stackrel{\cong}{\Longrightarrow}(X \cong Y)
$$

This needs a little explanation. The notation $f: X \stackrel{\cong}{\longrightarrow} Y$ (read as " $f$ is an equivalence") refers to a function such that for each $y$ in $Y$ the fiber $f^{-1}(y)$ is exactly one point. The notation $X \cong Y$ refers to the type $T$ of all equivalences between $X$ and $Y$. So the axiom refers to the natural function from the type $S$ of all equalities to the type $T$ of all equivalences.

There is a notion of $h$-level in univalent theory, which is the analogue of truncation level in homotopy theory, and is similar to the levels of homotopy $\lambda$-calculus. A type has $h$-level 0 if it has exactly one element; $h$-level 1 means that the type has at most one element, and is called a proposition. Informally, a proposition is a type which is "false" if it is empty, and "true" if it has one element, but this assumes the Law of the Excluded Middle; in an intuitionistic framework, a proposition need not be true or false. Inductively, a type $X$ has $h$-level $n+1$ if the type $x=x^{\prime}$ in $X$ has $h$-level $n$ for all $x, x^{\prime}$ in $X$. Thus $X$ has $h$-level 2 if the types $x=x^{\prime}$ in $X$ are propositions for all $x, x^{\prime}$ in $X$; informally, a type of $h$-level 2 may be thought of as a (discrete) set, since $x=x^{\prime}$ is either true or false for all $x, x^{\prime}$ in $X$-at least, assuming the Law of the Excluded Middle. The reader may enjoy interpreting $h$-levels of 3 or more.

In 2012-13, there was a special year at IAS on Univalent Foundations and type theory. This led to a resurgence in Voevodsky's work, in which many results have been formalized in Coq using the UniMath library; see [V15b].

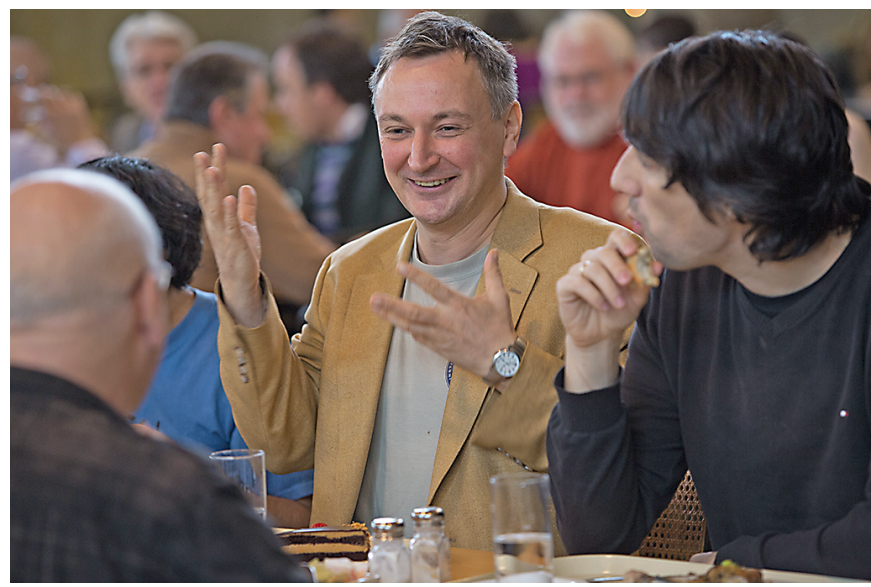

Voevodsky in 2013.

We mention two examples of papers using this technique. One is the paper [PVW], which formally constructs the $p$ adic numbers using a univalent approach, using Coq to verify the proof.

A second is the paper [ALV], where the authors compare three of the many algebraic structures that have been used for modeling type theory: categories with families, split type-categories, and representable maps of presheaves. If one assumes the univalence axiom, these notions can be meaningfully compared on the level of types; this contrasts with the situation in set theory, where one would need to resort to a comparison on the level of categories.

After 2013, Voevodsky worked primarily with contextual categories (also known as $C$-systems); the articles [V15, V16a, V16b,V17a,V17b] all deal with $C$-systems. One conjectures that $C$-systems can also be compared with the above structures.

When he passed away, Voevodsky left about 8 more preprints in various stages of completion, which begin to set up a formal framework in which one can prove the soundness of his univalent approach. Univalent foundations is now a subject in its own right, and Voevodsky's creation of this subject is a monumental achievement.

\section{References}

[AKS] Ahrens B, Kapulkin K, Shulman M. Univalent categories and the Rezk completion, Math. Str. Comp. Sci. (25): 1010-1039. MR3340533

[ALV] Ahrens B, Lumsdaine P, Voevodsky V. Categorical structures for type theory in univalent foundations, Computer science logic 2017, Art. No. 8, 16 pp., LIPIcs. Leibniz Int. Proc. Inform., 82, Schloss Dagstuhl. Leibniz-Zent. Inform., Wadern, 2017. MR3695532

[AwB] Awodey S, Bauer AJ. Propositions as [Types], Journal of Logic and Computation (14): 447-471, 2004. MR2081047 
[Ayb] Ayoub J. Les six opérations de Grothendieck et le formalisme des cycles évanescents dans le monde motivique. I,II. (French), Astérisque Nos. 314 and 315, 2007. MR2423375, MR2438151

[B80] Bloch S. Lectures on algebraic cycles, Duke University Math. Series, IV, Duke University Math. Department, Durham, N.C., 1980. MR558224

[B86] . Algebraic cycles and higher K-theory, Adv. in Math. (61): 267-304, 1986. MR852815

[D] Deligne P. Voevodsky's Lectures on Motivic Cohomology 2000/2001, Abel Symp. 4, Springer, Berlin, 355-409, 2009. MR2597743

[FV] Friedlander EM, Voevodsky V. Bivariant cycle cohomology, Cycles, transfers, and motivic homology theories: 138187, 2000. MR1764201

[G] Gersten S. Homotopy theory of rings, J. Algebra (19): 396-415, 1971. MR0291253

[HW] Haesemeyer C, Weibel C. The Norm Residue Theorem in Motivic Cohomology, Annals of Math. Studies, Princeton U. Press, 2019

[KV1] Voevodskiǐ VA, Kapranov MM. $\infty$-groupoids as a model for a homotopy category (Russian), Uspekhi Mat. Nauk (45): 183-184 (1990). MR1084995

[KV2] Voevodskii VA, Kapranov MM. The free $\boldsymbol{n}$-category generated by a cube, oriented matroids and higher Bruhat orders (Russian), Funktsional. Anal. i Prilozhen. (25): 62-65, 1991. MR1113124

[KV3] Kapranov MM, Voevodsky VA. Combinatorialgeometric aspects of polycategory theory: pasting schemes and higher Bruhat orders (list of results), International Category Theory Meeting (Bangor, 1989 and Cambridge, 1990). Cahiers Topologie Géom. Différentielle Catég. (32): 11-27, 1991. MR1130400

[KV4] Kapranov MM, Voevodsky VA. $\infty$-groupoids and homotopy types, International Category Theory Meeting (Bangor, 1989 and Cambridge, 1990). Cahiers Topologie Géom. Différentielle Catég. (32): 29-46, 1991. MR1130401

[Kato] Kato K. A generalization of local class field theory by using K-groups. II, J. Fac. Sci. Univ. Tokyo Sect. IA Math. (27): 603-683, 1980. MR603953

[M70] Milnor J. Algebraic $K$-theory and quadratic forms, Invent. Math. (9): 318-344, 1969/1970. MR0260844

[MV] Morel F, Voevodsky V. $\mathbb{A}^{1}$-homotopy theory of schemes, Inst. Hautes Études Sci. Publ. Math. (90): 45-143, 2001. MR1813224

[MVW] Mazza C, Voevodsky V, Weibel C. Lecture notes on motivic cohomology, Clay Mathematics Monographs, vol. 2, American Mathematical Society, Providence, RI, 2006. MR2242284

[MS82] Merkur'ev AS, Suslin AA. K-cohomology of SeveriBrauer varieties and the norm residue homomorphism, Izv. Akad. Nauk SSSR Ser. Mat. (46): 1011-1046, 1135-1136, 1982. MR675529

[OVV] Orlov D, Vishik A, Voevodsky V. An exact sequence for $K_{*}^{M} / 2$ with applications to quadratic forms, Ann. of Math. (165): 1-13, 2007. MR2276765
[PVW] Pelayo A, Voevodsky V, Warren M. A univalent formalization of the $p$-adic numbers, Math. Structures Comput. Sci. (25): 1147-1171, 2015. MR3340539

[Reh] Rehmeyer J. Voevodsky's Mathematical Revolution, Guest blog for Scientific American, 2013. Available at blogs. scientificamerican.com/guest-blog/voevodskye28099ssmathematical-revolution/

[Ro] Rost M. Chain lemma for splitting fields of symbols, 1998 preprint. Available at www.math.unibielefeld.de/ rost/chain-lemma.html

[ShV1] Shabat GB, Voevodsky VA. Drawing Curves over Number Fields, The Grothendieck Festschrift, Vol. III, 199-227, Progr. Math., 1990. MR1106916

$[\mathrm{ShV}]$. Equilateral triangulations of Riemann surfaces, and curves over algebraic number fields, Doklady Akad. Nauk SSSR (304): 265-268, 1989. MR0988486

[S00] Suslin A. Higher Chow groups and etale cohomology, Cycles, transfers, and motivic homology theories: 239-254, 2000. MR1764203

[SV96] Suslin A, Voevodsky V. Singular homology of abstract algebraic varieties, Invent. Math. (123): 61-94, 1996. MR1376246

[SV00a] . Relative cycles and Chow sheaves, $\mathrm{Cy}$ cles, transfers, and motivic homology theories: 10-86, 2000. MR1764199

[SV00b] B Bloch-Kato conjecture and motivic cohomology with finite coefficients, The Arithmetic and Geometry of Algebraic Cycles (Gordon, B. and Lewis, J. and MüllerStach, S. and Saito, S. and Yui, N., ed.), Nato ASI series C, vol. 548, Kluwer, 2000, pp. 117-189. MR1744945

[Vsh] Vishik A. Integral motives of quadics, PhD thesis, Harvard, 1997. MR2695910

[V90] Voevodskiï V. Étale topologies of schemes over fields of finite type over $\mathbb{Q}$. (Russian) Izv. Akad. Nauk SSSR Ser. Mat. (54): 1155-1167, 1990. MR1098621

[V91] Voevodskiï VA. Galois groups of function fields over fields of finite type over $\mathbb{Q}$. (Russian) Uspekhi Mat. Nauk (46): 163-164, 1991. MR1160339

[V91a] Voevodskiii VA. Galois representations connected with hyperbolic curves. (Russian) Izv. Akad. Nauk SSSR Ser. Mat. (55): 1331-1342, 1991. MR1152216

[V95] Voevodsky V. A nilpotence theorem for cycles algebraically equivalent to zero, Internat. Math. Res. Notices (4): 187-198, 1995. MR1326064

[V96] _. Homology of Schemes, Selecta Math. (2): 111153, 1996 MR1403354

[V98] . A $A^{1}$-homotopy theory, Documenta Mathematica, Proceedings of the ICM, Vol. I (Berlin, 1998): 579-604, 1998. MR1648048

[V99] . Voevodsky's Seattle lectures: $K$-theory and motivic cohomology, Notes by C. Weibel, Algebraic K-theory (Seattle, WA, 1997), Proc. Sympos. Pure Math., vol. 67, Amer. Math. Soc., Providence, RI, 1999, pp. 283-303. MR1743245

[V00a] Cohomological theory of presheaves with transfers, Cycles, transfers, and motivic homology theories: 87137, 2000. MR1764200 
[V00b] Triangulated categories of motives over a field, Cycles, transfers, and motivic homology theories: 188254, 2000. MR1764202

[V02a] Motivic cohomology groups are isomorphic to higher Chow groups in any characteristic, Int. Math. Res. Not. (7), 351-355, 2002. MR1883180

[V02b] Open problems in the motivic stable homotopy theory. I. Motives, polylogarithms and Hodge theory, Part I (Irvine, CA, 1998), 3-34, 2002. MR1977582

[V02c] - A possible new approach to the motivic spectral sequence for algebraic K-theory, Recent progress in homotopy theory (Baltimore, MD, 2000), 371-379, Contemp. Math., 293, Amer. Math. Soc., Providence, RI, 2002. MR1890744

[V03a] Motivic cohomology with Z/2-coefficients, Publ. Math. Inst. Hautes Études Sci. (98): 59-104, 2003. MR2031199

[V03b] . Reduced power operations in motivic cohomology, Publ. Math. Inst. Hautes Études Sci. (98): 1-57, 2003. MR2031199

[V04] . On the zero slice of the sphere spectrum, Tr. Mat. Inst. Steklova (246), 106-115, 2004. MR2101286

[V10a] Cancellation theorem, Documenta Math. (Extra volume): 671-685, 2010. MR2804268

[V10b] Motives over simplicial schemes, J. K-theory (5): 1-38, 2010. MR2600283

[V10c] - Motivic Eilenberg-Maclane spaces, Publ. Math. Inst. Hautes Études Sci. (112): 1-99, 2010. MR2737977

[V10d] . Simplicial radditive functors, J. K-theory, (5): 201-244, 2010. MR2640203

[V10e] - Unstable motivic homotopy categories in Nisnevich and cdh-topologies, J. Pure Appl. Algebra (214): 1399-1406, 2010. MR2593671

[V10f] Homotopy theory of simplicial sheaves in completely decomposable topologies, J. Pure Appl. Algebra (214): 1384-1398, 2010. MR2593670

[V11] On motivic cohomology with $\mathbb{Z} / l$ coefficients, Annals of Math. (174): 401-438, 2011. MR2811603

[V15] . A $C$-system defined by a universe category, Theory Appl. Categ. (30): 1181-1215, 2015. MR3402489

[V15b] An experimental library of formalized mathematics based on the univalent foundations, Math. Structures Comput. Sci. (25): 1278-1294, 2015. MR3340542

[V16a] Subsystems and regular quotients of $C$ systems, Contemp. Math. (658), Amer. Math. Soc., Providence, RI, 2016. MR3475277

[V16b] Products of families of types and $(\Pi, \lambda)$ structures on $C$-systems. Theory Appl. Categ. (31): 10441094, 2016. MR3584698

[V17a] C-systems defined by universe categories: presheaves, Theory Appl. Categ. (32): 53-112, 2017. MR3607209

[V17b] The $(\Pi, \lambda)$-structures on the $C$-systems defined by universe categories, Theory Appl. Categ. (32): 113121, 2017. MR3607210
[VRØ] Voevodsky V, Röndigs O, Østvær PA. Voevodsky's Nordfjordeid lectures: motivic homotopy theory, Motivic homotopy theory, 147-221, Universitext, Springer, Berlin, 2007. MR2334215

[VSF] Voevodsky V, Suslin A, Friedlander EM. Cycles, transfers, and motivic homology theories, Annals of Mathematics Studies, vol. 143, Princeton University Press, 2000. MR1764197

[W09] Weibel C. The norm residue isomorphism theorem, J. Topology (2): 346-372, 2009. MR2529300

Credits

Photos 1-3 are by Nadia Shalaby.

Photo 4 is by Cliff Moore, Institute for Advanced Study. Photo 5 is by Andrea Kane, Institute for Advanced Study.

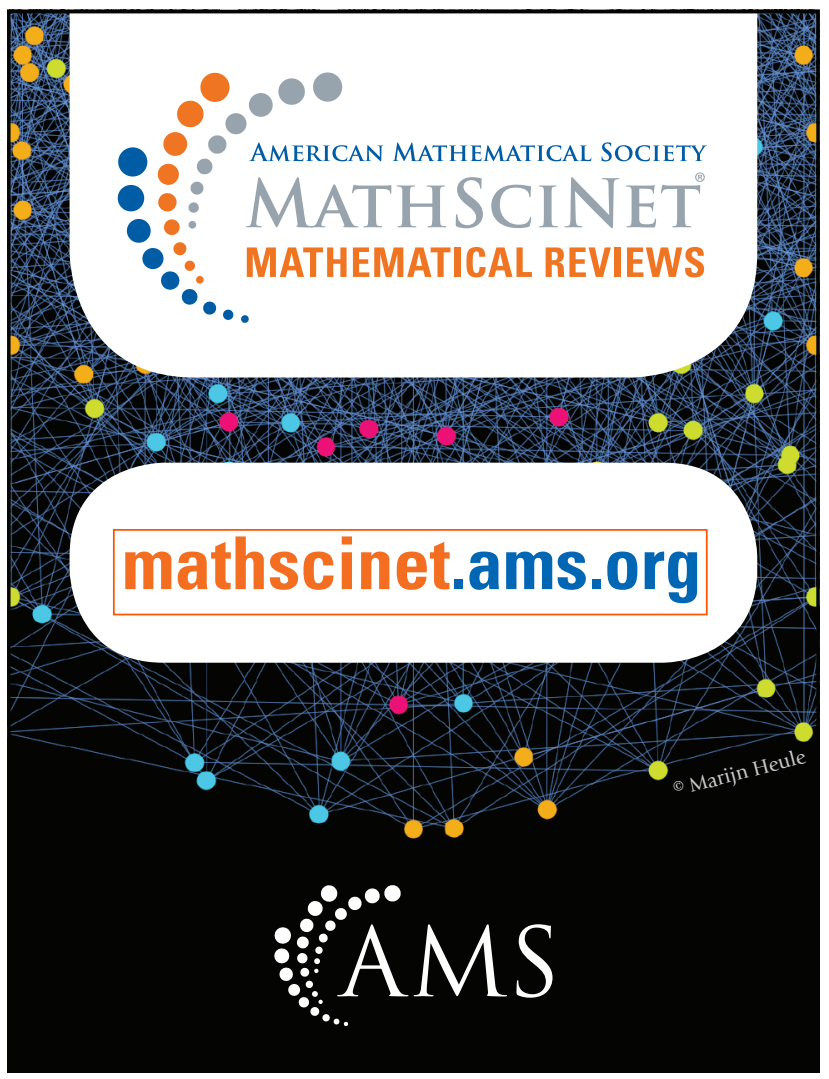

